\title{
COMPETENCIAS INVESTIGATIVAS DEL DOCENTE PARA EL FORTALECIMIENTO \\ DE SU PRAXIS PEDAGÓGICA
}

Kleeder José Bracho Pérez

pp 188-201

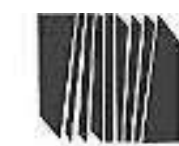

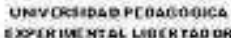

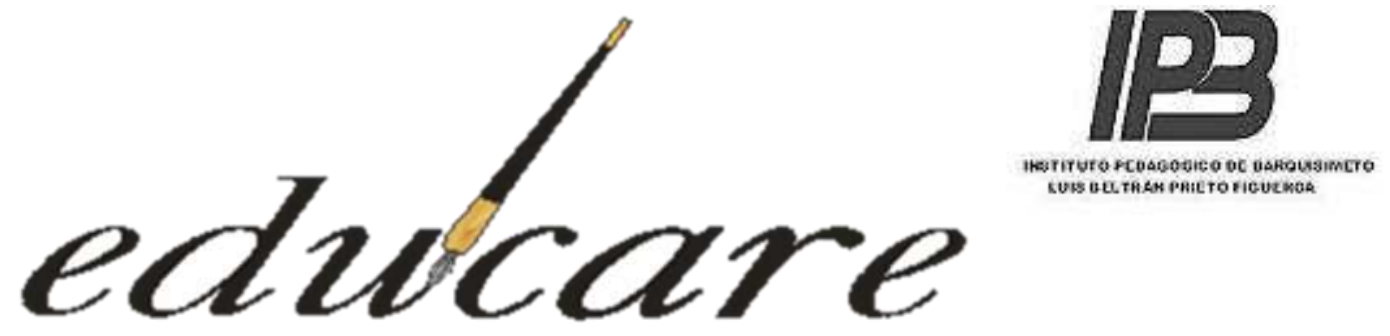

Órgano Divulgativo de la Subdirección de Investigación y Postgrado del Instituto Pedagógico de Barquasimeto "Luas Beltrán Prieto

Figueroa"

Volumen 23 № 1
COMPETENCIAS INVESTIGATIVAS DEL DOCENTE PARA EL FORTALECIMIENTO
DE SU PRAXIS PEDAGÓGICA
RESEARCH COMPETENCIES OF THE TEACHER FOR THE STRENGTHENING OF HIS
PEDAGOGICAL PRAXIS
I S S $N: 2244-7296$

\section{AUTOR}

Kleeder José Bracho Pérez

Universidad de Pamplona

\section{Colombia}




\title{
COMPETENCIAS INVESTIGATIVAS DEL DOCENTE PARA EL FORTALECIMIENTO DE SU PRAXIS PEDAGÓGICA
}

\author{
Kleeder José Bracho Pérez
}

pp 188-201

\section{Ensayo}

Recibido:07-01-2019

\section{RESUMEN}

Es necesario comprender que el desarrollo de las competencias investigativas en la praxis pedagógica, es un elemento esencial en el que va implícito la apropiación del conocimiento y el desarrollo potencial de habilidades, que despierta en el docente la capacidad de desempeñarse con altivez, de forma eficiente, eficaz y productiva, al vincular la teoría con la práctica y el abordaje del mismo en los diferentes ámbitos, sociales o laborales, que este se desempeña. En vista de lo planteado, el presente ensayo permite adentrarse en la investigación por los caminos transversales de la educación, donde se adquiere una decisión responsable, libre y permanente, que profundiza en los docentes los aspectos que determinan las competencia escriturales, discursivas, de análisis e interpretación, competencias que fortalecen los aspectos representativos de una cultura de la investigación, desde una óptica social y como actividad permanente de generación de conocimiento.

Palabras clave: Competencias; docente; praxis pedagógica.

\section{Kleeder José Bracho Pérez, \\ Universidad de Pamplona.}

Aceptado:03-04-2019

\section{ABSTRACT}

It is necessary to understand that the development of the investigative competencies in the pedagogical praxis, is an essential element in which the appropriation of the knowledge and the potential development of skills is implicit, that awakes in the teacher the capacity of To perform with pride, efficiently, efficiently and productively, by linking the theory with the practice and the approach of it in the different fields, social or labor, that this one works. In view of the above, this essay allows us to delve into research through the transversal paths of education, where a responsible, free and permanent decision is acquired, deepening in the teachers the aspects that determine the competence Writing, discourse, analysis and interpretation, competencies that strengthen the representative aspects of a culture of research, from a social perspective and as a permanent activity of knowledge generation.

Keywords: Competencies; teaching; pedagogical praxis.

* Licenciado en Educación, Mención Ciencias Pedagógicas, Área Tecnología Instruccional. Magíster en Gerencia Educativa. Doctor en Ciencias de la Educación. Docente de la Universidad de Pamplona, Pregrado y Postgrado e-mail: Kleeder.bracho@unipamplona.edu.co 


\section{COMPETENCIAS INVESTIGATIVAS DEL DOCENTE PARA EL FORTALECIMIENTO \\ DE SU PRAXIS PEDAGÓGICA}

Kleeder José Bracho Pérez

pp 188-201

\section{INTRODUCCIÓN}

En la contemporaneidad las distintas áreas del conocimiento han venido presentando un marcando cúmulo de características, que hacen resaltar los avances y la evolución abismal en los distintos escenarios en que el individuo le ha toca interactuar, contextos cargados de complejidad y determinados por la globalización que identifica los procesos dinámicos producidos por la sociedad en la que el hombre se encuentra sumergido.

Por esta razón, el docente está llamado a desarrollar su máximo potencial y apropiarse de competencias necesarias, que lo conlleven a desarrollar un mejor desempeño en la praxis educativa, permitiéndose apropiar de un contexto social con el que interactúa, para ayudar a proveerse de los conocimientos necesarios para la formulación de proyectos que contribuyan a generar soluciones propias y gestionadas a través de la investigación para poder determinar su productividad desde las aulas de clase hasta el ámbito social donde circunda su actividad laboral.

En tal sentido, se hace necesario que el docente se apropie de competencias que vayan más allá de su actividad rutinaria en el aula, que sea capaz de afrontar la realidad que vive, a fin de dar viabilidad a los problemas que enfrenta desde su práctica pedagógica. El desarrollo de competencias de investigación, hacen desvelar los misterios o dificultades con el esfuerzo del conocimiento científico, por lo que se concibe un docente investigador, crítico, autocrítico capaz de analizar y enfrentar los fenómenos sociales, que lo llevan a desarrollar valores de calidad para la formación y el desenvolvimiento del ser humano. Por último, se hace notable que investigando desde las aulas de clase se enseña y aprende mejor, se asumen los conocimientos y se adquieren en un entorno integrado y crítico. 


\section{COMPETENCIAS INVESTIGATIVAS DEL DOCENTE PARA EL FORTALECIMIENTO \\ DE SU PRAXIS PEDAGÓGICA}

Kleeder José Bracho Pérez

pp 188-201

\section{COMPETENCIAS DOCENTE ANTE EL CAMBIO}

Al hacer referencia al término de competencias, éste se encuentra estrechamente relacionado a las capacidades que posee el docente, destacándose así por sus conocimientos, habilidades, destrezas, pensamientos, carácter y valores, con que interactúa y se vincula en diferentes ámbitos, sociales o laborales, con el firme propósito de ser eficaz, eficiente y más productivo, para transformar la práctica pedagógica en la que se desenvuelve, para Spencer y Spencer (2003), consideran que es "una característica subyacente de un individuo, que está causalmente relacionada con un rendimiento efectivo o superior en una situación o trabajo, definido en términos de un criterio" (p. 9), competencia ha sido un término que en el campo educativo ha venido tomando más fuerza, afrontando situaciones específicas que involucran la creatividad, la innovación y la productividad.

El desarrollo de las competencias en el docente son demandadas en función de las necesidades y requerimientos que éste posee para desempeñarse en el contexto social y productivo de interacción, sin menos preciar otros sectores en los que puede estar inmerso, a nivel del desempeño o el tipo de actividad al que se dedica o incursiona, pues en este caso las competencias tienen el sentido de ser transferibles y específicas. Por su parte Alles (2005), "hace referencia a las características de la personalidad, devenidas de comportamientos que generan un desempeño exitoso en un puesto de trabajo" (p. 29). Sin embargo, cada cargo que el docente desempeña le exige características diferentes en cuanto a las competencias se refiere, estas deben ajustarse en el desenvolvimiento eficaz y eficiente dado en las necesidades de adecuación que son muy diversas.

Asimismo, estas competencias que el docente puede desarrollar desde su formación profesional y en las distintas áreas de estudio de la educación coadyuvan al proceso de formación integral y una vez finalizado este período, proporciona herramientas claves para 


\section{COMPETENCIAS INVESTIGATIVAS DEL DOCENTE PARA EL FORTALECIMIENTO \\ DE SU PRAXIS PEDAGÓGICA}

Kleeder José Bracho Pérez

pp 188-201

un desarrollo profesional exitoso. Por esta razón, es importante que el docente se acoja a los cambios adaptándose sin inconvenientes a las nuevas cualidades y habilidades competitivas que el sistema educativo requiere.

En este mismo orden de idea, los sistemas educativos han precisado la valoración de competencias claves en el docente, se ha constituido alrededor de elementos de acción fundacional inherentes a su accionar profesional, entre la práctica pedagógica y las escazas relaciones con las comunidades donde este interactúa. En torno a estos elementos, solo se han desarrollado pocas formas de apreciación o valoración del ejercicio docente, que van desde la evaluación de estudiantes, ejecución del Proyectos Educativos de aula e Integral (PEIC), con escaza profundidad y poco impacto, el cumplimiento de sus años de servicios para cumplir así un espacio contractual, dejando de lado el desarrollo de sus potencialidades que le garantice la apropiación de un conocimiento valido y útil, que lo identifique como un verdadero profesional de la educación. Bogoya (2000), señala que las competencia claves es "como el conjunto de rasgos que caracterizan al profesional" (p. 78).

Este tipo de competencias han sido de gran relevancia en un determinado tiempo y de un ineficiente esquema que constituye un modelo a seguir en los proceso de desempeño de actividades dentro de una institución educativa, el docente en la actualidad está llamado a renovar sus esquemas de comportamiento profesional integral, dotado de conocimientos pocos actualizados. Sobre esta base, se hace necesario que surja un profesional provisto de competencias específicas, bajo un enfoque protagónico que constituya un imperativo político para el desempeño de su labor docente con altos niveles de calidad.

Aunado a lo anterior el docente tienen un gran desafío ante sí mismo y la sociedad, éste debe enfrentar los cambios vertiginosos y radicales que a las sociedades les ha tocado atravesar y continuar con ese sentido misional de la educación, pero bajo la concepción de competencias específicas el cual constituye la formación de hombres desde una visión 


\section{COMPETENCIAS INVESTIGATIVAS DEL DOCENTE PARA EL FORTALECIMIENTO DE SU PRAXIS PEDAGÓGICA}

Kleeder José Bracho Pérez

pp 188-201

renovada holística e integral que el mundo amerita, este desempeño como práctica docente puede entenderse según la UNESCO (2005), como “el proceso de movilización de sus capacidades profesionales, su disposición personal y su responsabilidad social para: articular relaciones significativas entre los componentes que impactan la formación de los alumnos; participar en la gestión educativa; fortalecer una cultura institucional democrática, e intervenir en el diseño, implementación y evaluación de políticas educativas locales y nacionales, para promover en los estudiantes aprendizajes y desarrollo de competencias y habilidades para la vida" ( $\mathrm{p}$ 49) sumado a la construcción y gestión de conocimiento al servicio de dar respuestas a los problemas que se enfrenta.

Por otra parte, si se quiere tener una educación de calidad hay que invertir en un proceso de reingeniería del docente actual en función de sus competencias claves y específicas, consistiendo en gestionar y rediseñar los procesos de su práctica pedagógica logrando cambios radicales en su rendimiento medido a través de la innovación, las investigaciones y una mejora continua en su labor docente.

Para Tobón (2010), tienen un alto grado de especialización e implican procesos educativos específicos, se adquieren con la transmisión y asimilación, a partir de contenidos relativos a las áreas de estudio desde las distintas dimensiones del aprendizaje: el ser, conocer, hacer, y convivir; creando un individuo integral, del saber humanístico con habilidades de investigación, competencias que resultan necesarias para dominar un conocimiento y para después ser aplicado al área que practica.

Considerando lo expuesto anteriormente, se puede afirmar que son las competencias, en las que el docente debe apropiarse para el desempeño de su rol como noción central en el discurso sobre su práctica pedagógica, debe tener conocimientos claros y definidos, habilidades, destrezas, actitudes y valores que le consientan conocer la realidad social y educativa donde ejerce, e involucrarse efectiva y permanentemente a los procesos de investigación e innovación, su accionar institucional y social, considerando las estrategias 


\section{COMPETENCIAS INVESTIGATIVAS DEL DOCENTE PARA EL FORTALECIMIENTO \\ DE SU PRAXIS PEDAGÓGICA}

Kleeder José Bracho Pérez

pp 188-201

de aprendizaje como hipótesis de trabajo, para examinar y comprobar su eficacia, eficiencia y pertinencia, a fin de desarrollar la investigación desde su acción como elemento mediador, entre el conocimiento, el aprendizaje, las relaciones humanas que se generan en el contexto sociocultural y los roles que desempeña.

\section{COMPETENCIAS INVESTIGATIVAS DEL DOCENTE}

Las competencias investigativas son un elemento idóneo que emerge de una tarea concreta, en un contexto con sentido. Para López, Montenegro y Tapia (2005), la competencia investigativa está asociada siempre con algún ámbito del saber, por tanto se es competente o apto en acontecimientos en las que el conocimiento se orienta al juego. Bracho (2012), plantea la competencia en diferentes campos y caminos transversales de la educación, en donde se adquiere una decisión responsable así como libre y una educación permanente que profundiza los aspectos que cada uno determine.

Es ineludible que las competencias investigativa que asume el docente dentro de su práctica pedagógica posee implícito el desarrollo de habilidades y la apropiación del conocimiento, es decir, el docente debe tener la capacidad para desempeñar con altivez sus actividades y destrezas al vincular la teoría con la praxis investigativa, lo que le proporciona herramientas cognoscitivas, técnicas, metodológicas y procedimentales que lo conlleven a la observación compresión, análisis y reflexión crítica de la realidad entre el sujeto cognoscente y el objeto cognoscible. Por su parte, Beltrán (2006), señala que las competencias investigativa "constituyen herramientas mentales que permitirán conocer mejor la realidad y ser más razonables frente a ella", "saber cuándo y cómo actuar", "extraer significados de la experiencia vital que movilicen el desarrollo individual" (p. 3). 


\section{COMPETENCIAS INVESTIGATIVAS DEL DOCENTE PARA EL FORTALECIMIENTO \\ DE SU PRAXIS PEDAGÓGICA}

Kleeder José Bracho Pérez

pp 188-201

En el marco de las competencias investigativas para el fortalecimiento de la praxis pedagógica, se asume la investigación en la educación como la intención esencial del docente actual, contemporáneo, esta puede ser primordial para elaborar los diagnósticos de sus situaciones prácticas, abordada desde la reflexión sobre la acción permeado la realidad del aula y de los contextos en los que circula su práctica, del mismo modo, se plantean las competencia en diferentes campos que profundizan aspectos escriturales, discursivos, de análisis e interpretación.

\section{La Competencia Escritural}

Es necesario que el docente se apropie del hábito por la escritura, del cual requiere suma atención, coherencia, disciplina y organización, la redacción es una importante herramienta de comunicación que puede garantizar el éxito en todas sus actividades académicas que emprenda, el desarrollo de este tipo de competencia según López, Montenegro y Tapia (2005), la competencia escritural cuestiona la capacidad de escritura y actuación crítica y creativa en el planteamiento de opciones o alternativas generalizaciones y a proponer alternativas de explicaciones de un evento. Desarrollar la competencia escritural o producir textos de exposición y argumentarlos con coherencia y corrección gramatical. Contribuir a la formación holística en entornos académicos de los profesionales que puedan aportar, partiendo de sus saberes específicos, a la solución de los diversos problemas que presenta la sociedad con responsabilidad y alta sensibilidad social.

Aunado a lo anterior, esta competencia se debe desarrollar en los escenarios de acción del docente, el cual emplea factores cognoscitivos, socioculturales, sicológicos y lingüístico para describir mejor la realidad circundante. En este caso, Morales y Espinoza (2003), definen la competencia escritural como "una actividad intelectual en donde el docente se vale de sus experiencias, conocimientos y competencias para construir significados y reproducir ideas".(p 56) Este tipo de competencia se perfila como la base 


\section{COMPETENCIAS INVESTIGATIVAS DEL DOCENTE PARA EL FORTALECIMIENTO \\ DE SU PRAXIS PEDAGÓGICA}

Kleeder José Bracho Pérez

pp 188-201

principal para el desarrollo cognitivo del docente, generando las dinámicas que facilitan la reflexión, comprensión, la diferenciación y la diversidad textual.

Por otra parte, es necesario entender la competencia escritural es una actividad capaz de inquietar el interior del docente, visto como un acto liberador, realista, y crítico como mediación en la experiencia educativa, Peña (2007), concibe la competencia escritural como una aventura fascinante. Cuando se escribe como una aventura que mezcla la fascinación y el terror que se siente frente a una hoja en blanco, por lo que esta competencia no se deja enclaustrar en mapas ni esquemas mentales. Entonces se puede incidir que la desarrollar este tipo de competencia permitirá al docente seducir el campo del lenguaje y la comunicación.

\section{La Competencia Discursiva}

Esta hace énfasis en la necesidad de producir textos coherentes orales en determinados contextos sociales, es decir el docente debe desenvolver la lengua de manera eficaz bajo las composiciones gramaticales para un lograr producir un texto bien sea oral o escrito, a fin de abordar necesidades de comunicación, para Ortiz (1994), señala que la competencia discursiva es la capacidad que posee el docente para establecer una comunicación pedagógica efectiva y eficiente, al desarrollar en su personalidad un estilo flexible y lograr efectos educativos deseados, también considerada cuan variable de la comunicación interpersonal con efecto educativo social.

El docente que se apropia de la competencia discursiva se encuentra en total capacidad de abordar y nutrirse del conocimiento necesario para emplear la lengua de manera correcta bajo cualquier circunstancia, este tipo de competencia fija una línea en los proceso investigativos de la educación, otorgándole la habilidad de organizar ideas más allá de lo oracional, la comprensión del mensajes y la producción, donde la coherencia y cohesión juegan un papel sumamente importante. En consecuencia, poseer competencias discursivas 


\section{COMPETENCIAS INVESTIGATIVAS DEL DOCENTE PARA EL FORTALECIMIENTO \\ DE SU PRAXIS PEDAGÓGICA}

Kleeder José Bracho Pérez

pp 188-201

refiere, hablar fluidamente, con conexión y coherencia, lo que supone una correcta estructuración de ideas expresadas en un ordenamiento lógico.

En el caso de Van Dijk (2000), quien concibe la competencia discursiva como una manifestación de práctica, social y por ende cultural, el cual se desarrolla partiendo de la interacción entre los enunciados propios y ajenos; de ahí la presunción de usos discursivos dominantes y de usos de resistencia. Es decir, de ser una serie ordenada de palabras, códigos y proposiciones, también es una secuencia de actos recíprocamente relacionados, asimismo, el docente bajo la naturaleza interactiva y práctica de la lengua oral es capaz de participar en conversación y diálogos cotidianos y científicos.

\section{La Competencia Analítica}

Provee al docente la forma de experimentar y comprender de manera consiente cualquier situación o tema relacionado con su práctica pedagógica o situaciones propias, obteniendo orden en las ideas, coherencia, claridad y precisión entre otras cosas, este tipo de competencia ligada al pensamiento resumen el conjunto de actitudes autobservación, reflexión y toma de consciencia de las habilidades en el proceso de pensar, que el docente debe apropiarse y reconocer.

En este mismo orden de ideas, López, Montenegro y Tapia (2005), consideran que la competencia analítica es la capacidad de indagar la capacidad de explicar ideas, propuestas para la solución de un caso o un problema y se pide al sujeto evaluado que establezca la validez y pertinencia de los diferentes argumentos analizados o desglosados para escoger el mejor. (p. 103). En otras palabras, establece el diálogo auténtico y argumentativo al explicar las razones y los motivos que dan cuenta del sentido de lo que construye en texto.

Este tipo de competencia consiente al docente a la búsqueda de un razonamiento lógico que requiere convencer y persuadir. Este tipo de habilidad influye al docente a la explicación sobre cómo las diferentes partes de un proceso, se ordenan y se relacionan, para 


\section{COMPETENCIAS INVESTIGATIVAS DEL DOCENTE PARA EL FORTALECIMIENTO \\ DE SU PRAXIS PEDAGÓGICA}

Kleeder José Bracho Pérez

pp 188-201

lograr un efecto o conclusión; es decir, cuando se argumenta, se explica el porqué de las cosas, se justifica la ideas, se dan razones, por lo que se establecen sus propios criterios. Este tipo de competencia señala Hernández (2008), que consiste en sustentar o dar explicación de determinados procesos, proposiciones, artículos, planteamientos, procedimientos, teorías, sucesos, anécdotas, mitos y hasta fenómenos naturales y sociales. Sobre esto afirma Pérez (2000), asumir una perspectiva analítica, escritural y discursiva implica generar escenarios para la generación de conocimiento, unas herramientas teóricas, y definir los objetos de trabajo pedagógico.

\section{La Competencia Interpretativa}

Supone la generación de hipótesis, el establecimiento de conjeturas y la resolución de problemas. De igual manera, hace referencia a la construcción de modelos creativo y eficiente de estrategias de trabajo para López, Montenegro y Tapia (2005), la competencia interpretativa evalúa la capacidad de comprensión del texto, problema, esquema, gráfico o mapa y se pide al sujeto evaluado que decida cuál de las interpretaciones ofrecidas en las alternativas de respuestas es la mejor. (p. 103). En otras palabras, esta competencia permite explicitar el verdadero sentido de un texto y asumir posiciones críticas frente al mismo dominando las relaciones y los ejes significativos.

Esta competencia se refleja en las acciones inherentes al planteamiento de soluciones a situaciones problemas, en la generación de preguntas e hipótesis, la construcción de mundos posibles incitados por la interpretación de los textos escritos. En esta etapa el docente puede lograr ser productivo, trabajos de investigación bien definidos que le permite concretar su accionar pedagógico a través de la apropiación de dichas competencias interpretativa y argumentativa en los textos producidos por él, ya sea producto de una investigación concerniente a labor educativa, a la realidad inmediata de la que se enfrenta día a día. 


\section{COMPETENCIAS INVESTIGATIVAS DEL DOCENTE PARA EL FORTALECIMIENTO \\ DE SU PRAXIS PEDAGÓGICA}

Kleeder José Bracho Pérez

pp 188-201

El docente al apropiarse de estas competencias, desarrolla el gusto por la lectura, la cual le permite promover en él un pensamiento crítico de su entorno académico, laborar y cotidiano, lo transforma en un individuo autónomo capaz de comprender y valorar las diversas formas de abordar el conocimiento de asimilarlo y transmitirlo, poniéndolo en función de transformar el espacio y las prácticas pedagógicas en los momentos de sociabilidad y reflexiones críticas, para que sean interpretados de múltiples formas y generar un acercamiento a las posibilidades de cambio en la educación a través de la investigación, es de considerar además que las tecnologías de información y comunicación mantienen abierto sus espacios para el conocimiento, el aprendizaje y la producción al que tendrá acceso el docente que haya desarrollado dichas competencias.

\section{CONSIDERACIONES FINALES}

Resaltar las competencias investigativas del docente para el fortalecimiento de su praxis pedagógica, es una esencial particularidad del proceso de formación continua y autoformación, que el perfil profesional docente conviene desarrollar como punto que refuerza la perfección en los procesos educativos. Del mismo modo, es necesario desplegar la praxis de la investigación dentro de los programas formación profesional en el marco de una metodología integradora con un enfoque dirigido al mejoramiento de la calidad educativa.

El desarrollar competencias, con el propósito de promover la investigación en la práctica educativa, para consolidar en conocimiento el cual combinado con las experiencias accederá a delinear la producción científica abordada desde la perspectiva de la adquisición, fusión y la adaptación, manejables del conocimiento desde su ámbito de acción. 


\section{COMPETENCIAS INVESTIGATIVAS DEL DOCENTE PARA EL FORTALECIMIENTO \\ DE SU PRAXIS PEDAGÓGICA}

Kleeder José Bracho Pérez

pp 188-201

Aunado a lo anterior, el docente es un ente de proyección, capaz de formarse en función de necesidades del mundo contemporáneo reclama, considerando la investigación un reflejo de condiciones socio-culturales y políticas del mundo globalizado. Los docentes, se deben enfrentar en su práctica pedagógica con complejas exigencias que requieren políticas de cambio flexibles en la forma de apropiarse de competencias investigativas y darse oportunidades permanentes de auto aprendizaje.

\section{REFERENCIAS}

Alles, M. (2005). Dirección estratégica de recursos humanos. Ediciones Granica.

Andrade. R. (2.012). Universidad de Guanajuato. Accedido el 8 de Marzo, 2018 desde: www.Rena.edu.ve/docentes/articulos/competencias.pdf

Beltrán, S. (2006). Competencias investigativas. Ariel. España.

Bogoya, D.(2000). El Debate Sobre Las Competencias. Magisterio Colombia.

Bracho, K. (2012). Cultura Investigativa y Producción Científica en Universidades Privadas del Municipio Maracaibo del Estado Zulia. REDHECS: Revista electrónica de Humanidades, Educación y Comunicación Social, ISSN-e 1856-9331, Año 7, No. 12, 2012, págs. 50-69.

Hernández, E. (2008). Competencias investigativas del docente en las universidades las bases de datos Pubmed e I.M.E. Universidad de Murcia. Ciencias

López, L.; Montenegro, M. y Tapia, R. (2005). La investigación, eje fundamental en la enseñanza del derecho. Guía práctica. Colombia. Publicaciones de la Universidad Cooperativa de Colombia. 


\section{COMPETENCIAS INVESTIGATIVAS DEL DOCENTE PARA EL FORTALECIMIENTO \\ DE SU PRAXIS PEDAGÓGICA}

Kleeder José Bracho Pérez

pp 188-201

Morales, O. y Espinoza, N. (2003). Lectura y escritura: coexistencia entre lo impreso y lo electrónico. Educere. Vol 7, No. 022. Venezuela. Redalyc. Lectura y escritura: coexistencia entre lo impreso y lo electrónico. Educere.

Ortiz, E. (1994). "El perfeccionamiento del estilo comunicativo del maestro para su labor pedagógica". Lenguaje y Textos 8 Universidad de la Coruña.

Peña, L. (2007). ¿Qué significa escribir? Bogotá: Periódico Altablero, MEN. Accedido el 4 de Marzo, 2018, desde http://www.mineducacion.gov.co/1621/article-122242.html

Pérez, A. (2000). Hacia una pedagogía del discurso: elementos para pensar la competencia argumentativa en los procesos de escritura en la educación básica. Competencias y proyectos pedagógicos. Bogotá: Universidad Nacional de Colombia

Spencer, L. y Spencer, S. (2003). Competencias en el trabajo. Modelos para un rendimiento superior. Papers de formación Municipal, Número 57.

Tobón, S. (2006), Competencias, calidad y educación superior. Alma mater colección. Editor Cooperativa Editorial Magisterio. Colombia.

UNESCO. (2005), Proyecto Regional de Educación para América Latina y el Caribe (PRELAC).

Van Dijk, T. A. (2000). El discurso como interacción social. Estudios sobre el discurso II. Una introducción multidisciplinaria. Barcelona: Gedisa. 